\title{
Lane-Based Optimization for Macroscopic Network Configuration Designs
}

\author{
C. K. Wong and Yi Liu \\ Department of Architecture and Civil Engineering, City University of Hong Kong, Tat Chee Avenue, Kowloon Tong, Hong Kong \\ Correspondence should be addressed to C. K. Wong; wongck@cityu.edu.hk
}

Received 9 May 2017; Accepted 13 June 2017; Published 19 July 2017

Academic Editor: Stefan Balint

Copyright ( 2017 C. K. Wong and Yi Liu. This is an open access article distributed under the Creative Commons Attribution License, which permits unrestricted use, distribution, and reproduction in any medium, provided the original work is properly cited.

\begin{abstract}
Lane markings (arrows) at individual intersections serve as interfaces to connect upstream and downstream intersections in signal-controlled networks. Demand flows from origins to destinations may need to pass through a series of intersections. If lane markings are not well established to ban turns at intersections, then paths connecting origin and destination (OD) pairs could be inefficient. Due to indirect connections, road users need to take longer paths to reach their destinations. Conventionally, network configurations are fixed inputs for network analysis. In the present study, concepts of the lane-based designs for individual signalized intersections are extended for signal-controlled network designs. Taking OD demand flows as inputs, the proposed algorithm will optimize all lane markings and assigned lane flows on approach lanes. Paths (flows) will then be optimized by linking up the optimized lane markings across upstream and downstream intersections. Traffic signal settings at individual intersections will be optimized simultaneously by maximizing the reserve capacity for the entire OD demand flow matrix. The problem is formulated as a Binary-Mixed-Integer-Linear-Program (BMILP) and a standard branch-and-bound routine is applied to solve for global optimum solutions. A numerical example using a 4-intersection network will be given to demonstrate the effectiveness of the proposed design methodology.
\end{abstract}

\section{Introduction}

In the optimization of signal-controlled networks, users' travel routings are represented by path flows and link flows. Travel patterns from different origin and destination pairs through different paths could be influenced by traffic signal settings at individual signalized intersections and the network link connectivity. Network link connections are depending on the lane marking patterns. For network design problems, different formulations and solution methods have been developed to solve this complex multilevel programming problem adopting different design objectives, model assumptions, and application conditions [1-4]. A comprehensive literature review on the road network design is given [5]. Traffic signal coordination incorporating a path-based assignment algorithm using a TRANSYT traffic model was optimized [6]. Signalized Cell Transmission Model (CTM) has been developed and applied to optimize the coordinated signal settings for a pair of adjacent traffic signals to avoid spillback of spatial queues and preventing gridlock due to lane closure for work zone pairs $[7,8]$. All of these methods assumed that the network configurations and lane markings are given as fixed exogenous inputs. Based on the given set of lane markings, grouping traffic lanes into traffic streams to form a network link is performed manually and traffic signal settings can then be optimized by using either stage- or phase- (group-) based methods [9]. Stage-based designs of traffic signal settings were found to influence significantly the optimized network performances [10-14]. Phase- or groupbased designs of traffic signal settings were found in previous studies $[6,15,16]$. Pregrouping traffic lanes into traffic streams to form network links to generate network configuration for signalized network design analysis may not be optimal to match the demand flow patterns. We would like to relax the lane markings as design variables in the proposed lane-based design method to further enhance the network link groupings and connectivity taking the latest lane-based design concept into considerations.

Extending green duration times for the heaviest traffic stream may allow more discharges to reduce congestion. 
However, it may not be easily achieved as other conflicting traffic streams may then be suffering from loss of green times within a signal cycle. It may increase total delay and cause oversaturation. However, if adjacent traffic lanes occupied by other less critical turn movements with spare capacities are designed with proper lane markings, heavy traffic loadings could be evenly distributed onto approach traffic lanes. Discharge rates of the traffic streams can be increased even without extending their green duration times in a signal cycle so that conflicting traffic streams from other arms will not be adversely affected. This improvement scheme is attractive as it may be simply achieved by altering the lane marking patterns. Entire network connectivity would be improved. This design concept has been successful in designing individual signalized intersections [17]. Intersection capacity has been increased significantly if lane markings and traffic signal settings are optimized in a unified framework [18-21].

In network design problems, we should ensure that feasible paths exist to connect all origin-destination (OD) demand flow pairs according to users' inputs. If the link connectivity to form a network configuration is not well designed, probably only one congesting path exists to carry the heaviest OD demand flow alone while other network links may not be fully utilized. This is possible due to fixed inputs of lane markings in conventional network designs. Indeed, optimizing the lane marking patterns across adjacent intersections could generate more feasible paths for users' route choices in traveling through a network. OD demand flows could be assigned onto these feasible paths to relieve the network congestion. The proposed methodology will be able to optimize the network configuration through optimizing the individual lane markings on approach lanes at intersection level so as to improve the overall network connectivity. It is expected that multiple OD demand flows of asymmetrical flow intensities could also be tackled. More importantly, the proposed algorithm is possible to eliminate unnecessary lane markings which may lead to redundant paths to connect some noncritical OD pairs. Overall network efficiency is improved. To optimize the control efficiency, reducing the number of conflicting movements at intersections and then reducing the loss times within a signal cycle are the new model features. Numerical example will be given to demonstrate the enhancement of lane usage through establishing proper lane markings at intersection levels to improve the link connectivity and network configuration serving the assigned flow patterns for a signal-controlled network. Usual design objective to maximize the reserve capacities of all involved intersections will be adopted for optimization. The problem is a Binary-Mixed-Integer-Linear-Program (BMILP) that could be effectively solved by standard branch-and-bound routines.

\section{Constraints for Connecting Network Links}

\subsection{Assigning Given OD Demand Flows into Path Flows}

$$
\mu T_{a, b}=\sum_{h_{a, b}=1}^{\bar{H}_{a, b}} P_{a, b, h_{a, b}}, \quad \forall a \in A, b \in B,
$$

where $a$ and $b$ are, respectively, the origin and destination (nodes) in a study signal-controlled network, $A$ and $B$ are, respectively, the total numbers of origin and destination (nodes), $\mu$ is a common flow multiplier to scale the given demand flows, $h_{a, b}$ is the path number connecting origin $a$ and destination $b, \bar{H}_{a, b}$ is the total number of paths to connect origin $a$ and destination $b, T_{a, b}$ is the given demand flow from origin $a$ to destination $b$ that should equal the sum of all path flows, and $P_{a, b, h_{a, b}}$ is the path flow from origin $a$ to destination $b$ using the $h_{a, b}$ path. In Figure 1 , for example, there is an OD pair connecting origin $a$ and destination $b$ with 3 paths: $h_{a, b}-1, h_{a, b}$, and $h_{a, b}+1$. If $T_{a, b}=1,000 \mathrm{pcu} / \mathrm{h}$, then numerically $1,000 \mathrm{pcu} / \mathrm{h}$ should equal the sum of path flows for conservation, $P_{a, b, h_{a, b}-1}+P_{a, b, h_{a, b}}+P_{a, b, h_{a, b}+1}$.

\subsection{Existence of a Path Connecting an OD Pair}

$$
\begin{aligned}
M^{2} P_{a, b, h_{a, b}} \geq M \alpha_{a, b, h_{a, b}} \geq & P_{a, b, h_{a, b}}, \\
& \forall a \in A, b \in B, h_{a, b} \in H_{a, b},
\end{aligned}
$$

where $\alpha_{a, b, h_{a, b}}$ is a binary variable to denote the existence of the $h_{a, b}$ path connecting origin $a$ and destination $b, P_{a, b, h_{a, b}}$ is a path flow from origin $a$ to destination $b$ using the $h_{a, b}$ path, and $M$ is an arbitrary large number (e.g., 1000). Referring to the example given in Figure 1, if $P_{a, b, h_{a, b}-1}=600 \mathrm{pcu} / \mathrm{h}, P_{a, b, h_{a, b}}$ $=400 \mathrm{pcu} / \mathrm{h}$, and $P_{a, b, h_{a, b}+1}=0 \mathrm{pcu} / \mathrm{h}$, then correspondingly $\alpha_{a, b, h_{a, b}-1}=\alpha_{a, b, h_{a, b}}=1$ and $\alpha_{a, b, h_{a, b}+1}=0$. Paths $h_{a, b}-1$ and $h_{a, b}$ exist for connecting the OD pair from origin $a$ and destination $b$ but path $h_{a, b}+1$ does not exist.

\subsection{Demand Turning Flows at Intersections from Path Flows of} Different OD Pairs. In the present formulation, users' given OD demand flows are model inputs. Inside a study signalcontrolled network, multiple paths may be available for connecting an OD pair. These path flows may pass through different intersections from $\operatorname{arm} i$ to $\operatorname{arm} j$ at intersection $n$. For an intersection $n$, turning flow from arm $i$ to $\operatorname{arm} j$ may involve different OD pairs depending on their used paths. Therefore, the actual demand turning flows at intersections (from arm $i$ to arm $j$ making a turn) would be the sum of these involved path flows. Since we do not explicitly consider traffic signal coordination in the present study, demand arrivals obtained from the path flows are assumed to reach all intersections spontaneously.

In Figure 2, for example, there are two origins $a$ and $a^{\prime}$ and one destination $b$. For a typical intersection $n=12$, there are 4 arms $i=j=\{1,2,3,4\}$. A left-turn movement could be denoted by $(n, i, j)=(12,1,2)$ or $(12,2,3)$. From the two origins, suppose 4 paths exist to enter destination $b$. At intersection $n=25$ from arm $i=4$ to $\operatorname{arm} j=3$, it is a right-turn movement. 3 path flows make this right-turn. Thus, demand flow $Q_{n, i, j}$ should be the sum of the 3 path flows $P_{a, b, h_{a, b}}, P_{a, b, h_{a, b}+1}$, and $P_{a^{\prime}, b, h_{a^{\prime}, b}}$. In general, to model all 


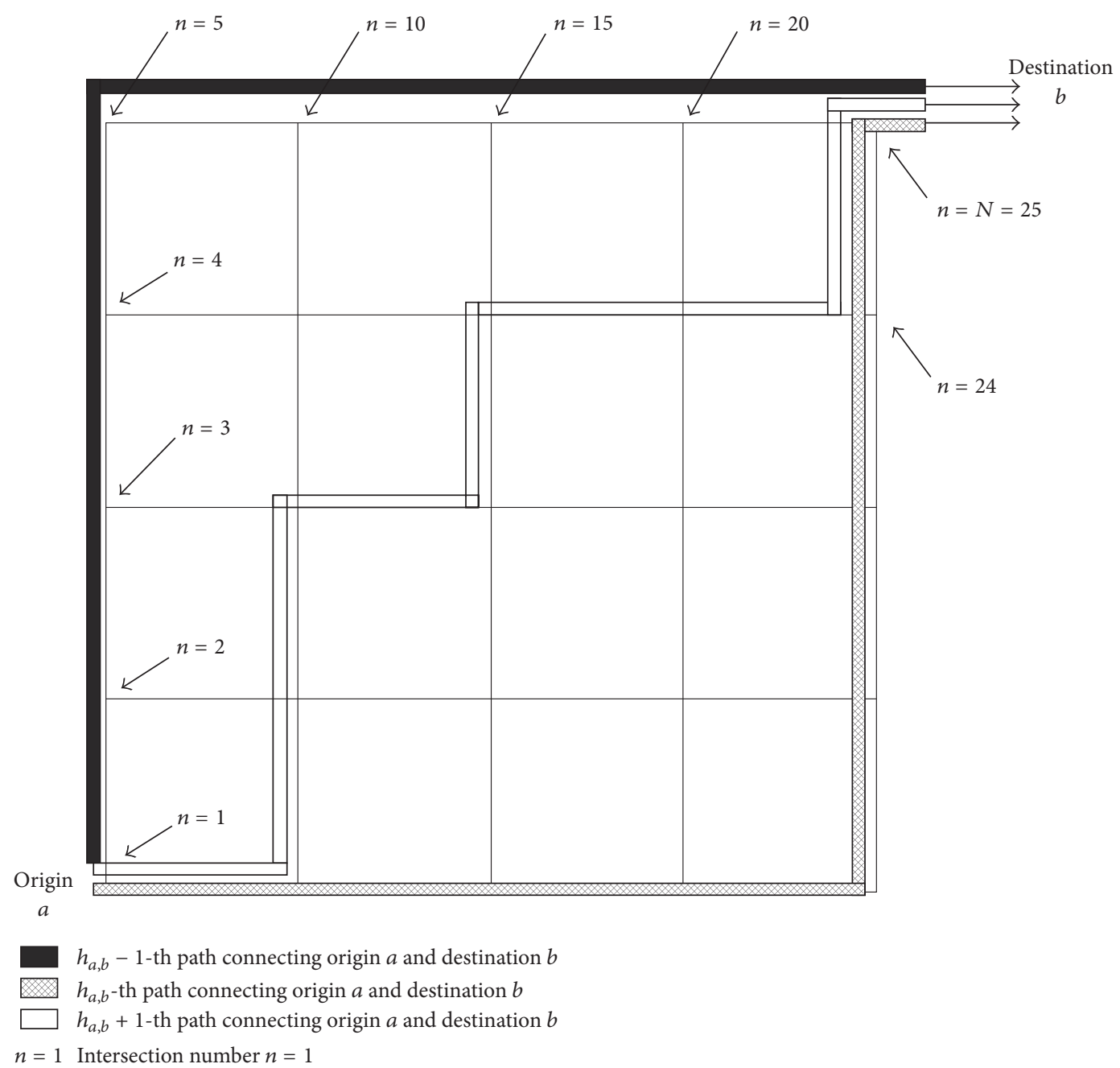

FIgURE 1: Paths connecting OD pairs in an example signal-controlled network.

paths and all OD pairs, we need the following constraint set to evaluate the demand turning flows at intersections

$$
Q_{n, i, j}=\sum_{a=1}^{A} \sum_{b=1}^{B} \sum_{h_{a, b}=1}^{\bar{H}_{a, b}} P_{a, b, h_{a, b}}, \quad \forall\left(a, b, h_{a, b}\right) \in F(n, i, j),
$$

where $F(n, i, j)$ is a mathematical function to identify the path $h_{a, b}$ from origin $a$ to destination $b$ when $(n, i, j)$ are given as inputs.

\subsection{Existence of OD Demand Flows}

$$
M \beta_{a, b} \geq T_{a, b} \geq \beta_{a, b}, \quad \forall a \in A ; b \in B,
$$

where $\beta_{a, b}$ is a binary-type variable to denote whether OD demand flow from origin $a$ to destination $b$ exists. When OD flow $T_{a, b}$ is nonzero as given by users, $\beta_{a, b}$ should equal "1." $M$ is an arbitrary positive number.

2.5. Existence of Path Flows for Connecting OD Pairs. From Section 2.4, when OD demand flow $T_{a, b}$ exists with nonzero user's input, then a binary variable $\beta_{a, b}$ should equal " 1 " numerically. Whenever $\beta_{a, b}=1$, at least one path should exist to serve the demand flow as governed by (5). For heavy OD demand flows, multiple paths may exist to share the loadings in different network links. Equation (5) may allow the existence of multiple paths $\left(\alpha_{a, b, h_{a, b}}>1\right)$ when $\beta_{a, b}=1$.

$$
\sum_{h_{a, b}=1}^{\bar{H}_{a, b}} \alpha_{a, b, h_{a, b}} \geq \beta_{a, b}, \quad \forall a \in A ; b \in B,
$$

where $\alpha_{a, b, h_{a, b}}$ is a binary variable to denote the existence of the $h_{a, b}$ path connecting origin $a$ and destination $b$ and $\beta_{a, b}$ is a binary-type variable to denote the existence of an OD flow from origin $a$ to destination $b$.

Also from Section 2.4, if users give zero flow input for an OD pair, $\beta_{a, b}$ is forced to be zero numerically. And it in turn forces $\alpha_{a, b, h_{a, b}}$ to be zero. No path should exist as governed by

$$
\beta_{a, b} \geq \alpha_{a, b, h_{a, b}}, \quad \forall a \in A ; \forall b \in B ; \forall h_{a, b}=1, \ldots, \bar{H}_{a, b} .
$$




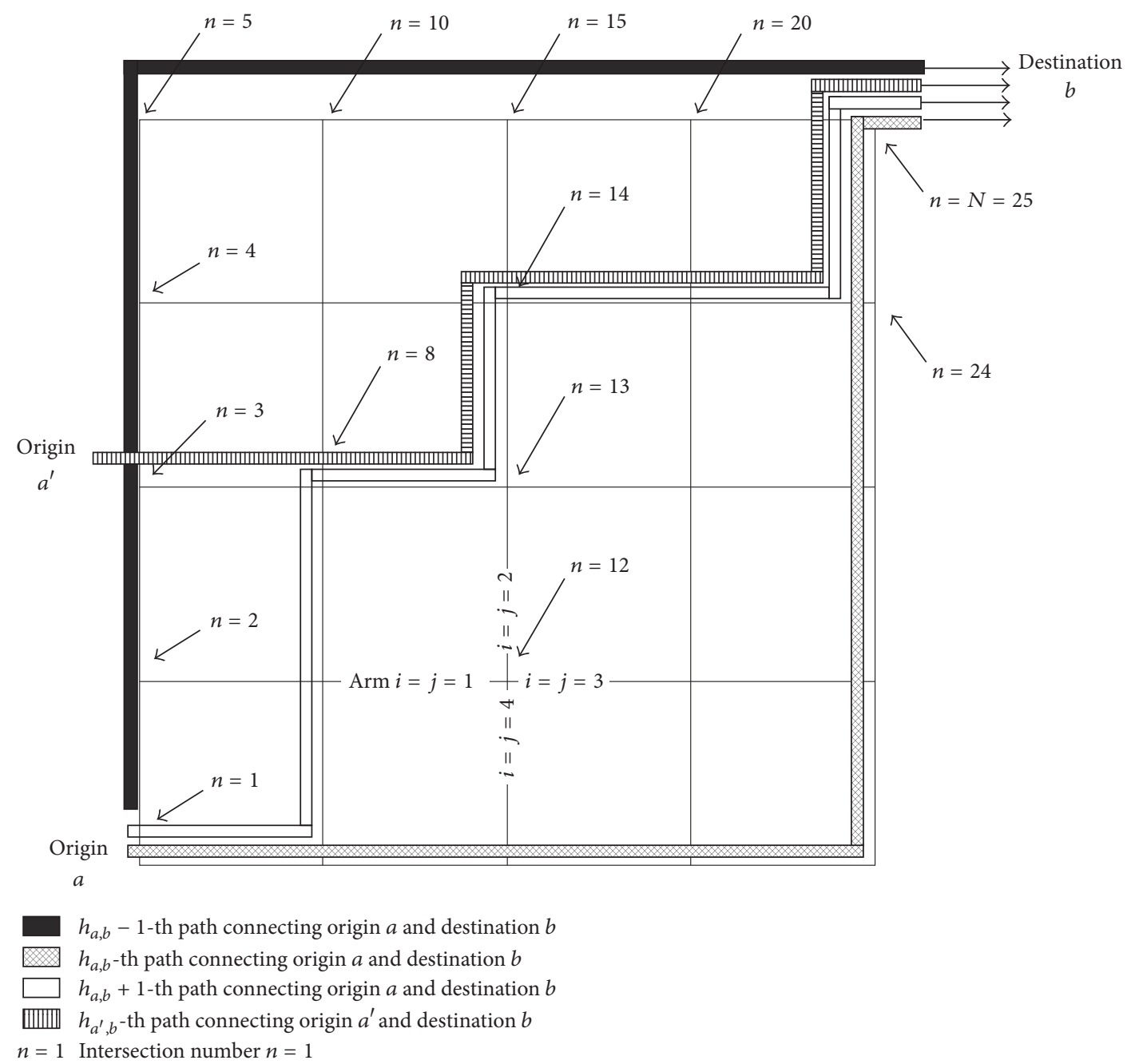

FIGURE 2: Converting path flows to turning flows at intersections for all OD pairs.

\subsection{Establishing Lane Markings at Intersections to Form a Path Connecting OD Pairs}

$$
\sum_{k} \delta_{n, i, j, k} \geq \alpha_{a, b, h_{a, b}}, \quad \forall(n, i, j, k) \in F^{\prime}\left(a, b, h_{a, b}\right)
$$

where $\delta_{n, i, j, k}$ is a binary-type variable to denote the existence of a lane marking permitting a turn from arm $i$ to arm $j$ on lane $k$ at intersection $n$ and $\alpha_{a, b, h_{a, b}}$ is a binary variable to model the existence of the $h_{a, b}$ path connecting origin $a$ and destination $b$. Whenever a path exists, $\alpha_{a, b, h_{a, b}}=1$, associated lane markings for turns at intersections must exist on approach lane $k$ so that path $h_{a, b}$ can be established to connect origin $a$ and destination $b$. And $F^{\prime}\left(a, b, h_{a, b}\right)$ is a user defined mathematical function to identify all movement turns from arm $i$ to arm $j$ on lane $k$ at intersection $n$ for path $h_{a, b}$ connecting origin $a$ and destination $b$.

2.7. Prohibiting Lane Markings for Nonexistence of Destination. Depending on users' OD demand flow inputs, some nodes could be serving as origins but not as destinations. Therefore, corresponding turns entering these nodes at various intersections may be unnecessary. Respective lane markings should be prohibited for existence

$$
\sum_{a=1}^{A} \sum_{h_{a, b} \in H_{a, b}^{\prime}} \alpha_{a, b, h_{a, b}} \geq \sum_{k=1}^{L_{n, i}} \delta_{n, i, j, k}, \quad \forall b \in B,
$$

where $\alpha_{a, b, h_{a, b}}$ is a binary variable to denote the existence of the $h_{a, b}$ path connecting origin $a$ and destination $b$. And only those $h_{a, b} \in H_{a, b}^{\prime}$ are considered $\left(H_{a, b}^{\prime} \subset H_{a, b}\right)$ as paths from the set $H_{a, b}^{\prime}$ will utilize the same lane marking to turn to the same destination node $b$ from different origins $a$. In Figure 2, assume 3 paths exist to connect origin $a$ and destination $b$ in which two of them $h_{a, b}$ and $h_{a, b}+1$ require a right-turn lane marking to enter destination $b$ at intersection $n=25$. Therefore, the set relationships may be given as follows: $\left(h_{a, b}, h_{a, b}+\right.$ 1) $\in H_{a, b}^{\prime} \subset H_{a, b}$. With respect to users' input demand flows for this OD pair, it may be possible that all demand flows are assigned to the path $h_{a, b}-1$ only and thus the corresponding path flows for the other two paths do not exist; that is, $\alpha_{a, b, h_{a, b}}=\alpha_{a, b, h_{a, b}+1}=0$. Besides, from another origin $a^{\prime}$, its 
path to reach the destination $b$ also requires a right-turn lane marking at intersection $n=25$. If the path flow for this OD pair is also zero, then the corresponding $\alpha_{a^{\prime}, b, h_{a^{\prime}, b}}=0$. If similar zero path flow is found for all origins to enter this destination $b$ using the right-turn lane marking $(n, i, j)=(25,4,3)$, this right-turn lane marking then becomes unnecessary and thus the lane marking variable $\delta_{n, i, j, k}$ on all approach lane $k$ (taking summation) should be prohibited. Mathematically, we need a user defined function $F^{\prime}\left(a, b, h_{a, b}\right)$ to identify all intersections $n$ and their corresponding turns from arm $i$ to $\operatorname{arm} j,(n, i, j)=F^{\prime}\left(a, b, h_{a, b}\right)$ for entering destination $b$.

2.8. Prevention of Unnecessary Lane Markings. Having restricted those unnecessary lane markings at intersections near a destination node whenever path flows are zero as given in Section 2.7, there may still exist some other useless lane markings at other intersections where arms are not directly connecting to destination nodes. Those lane markings are not serving any path flow which should be restricted from existence. The following constraint set is developed so that these useless lane markings could all be prohibited:

$$
\Delta_{n, i, j, a, b, h_{a, b}}=\alpha_{a, b, h_{a, b}}, \quad \forall(n, i, j)=F^{\prime}\left(a, b, h_{a, b}\right),
$$

where $\alpha_{a, b, h_{a, b}}$ is a binary variable to denote the existence of the $h_{a, b}$ path connecting origin $a$ and destination $b$ and $\Delta_{n, i, j, a, b, h_{a, b}}$ is an auxiliary binary variable to identify the turn from arm $i$ to arm $j$ at intersection $n$ to support the $h_{a, b}$ path connecting origin $a$ and destination $b$. In Figure 2, for example, from origin $a$ to destination $b$ along the $h_{a, b}$ path, at intersection $n=1$, traffic flows should be turning from $\operatorname{arm} i=1$ to arm $j=3$ and thus $\Delta_{n=1, i=1, j=3, a, b, h_{a, b}}=1$. Similarly, traffic flows should be turning from arm $i=4$ to arm $j=2$ at intersection $n=24$ and thus $\Delta_{n=24, i=4, j=2, a, b, h_{a, b}}$ $=1$. More importantly, at the same intersection $n=24$, no path flow turns left from arm 4 to arm 1 and therefore $\Delta_{n=24, i=4, j=1, a, b, h_{a, b}}=0$. Again, the user defined mathematical function $F^{\prime}\left(a, b, h_{a, b}\right)$ is to identify all turns from arm $i$ to arm $j$ at intersection $n$ with $(n, i, j)=F^{\prime}\left(a, b, h_{a, b}\right)$.

With the auxiliary variable $\Delta_{n, i, j, a, b, h_{a, b}}$, we can determine whether a specific lane marking $\delta_{n, i, j, k}$ turning from arm $i$ to $\operatorname{arm} j$ at intersection $n$ is required or not. The lane marking is considered to be useless if all path flows from all OD pairs do not require it along all approach lanes. The following constraint set is then introduced to prevent all useless lane markings from existence in the optimization process

$$
\begin{array}{r}
M^{2} \sum_{k=1}^{L_{n, i}} \delta_{n, i, j, k} \geq M \sum_{a=1}^{A} \sum_{b=1}^{B} \sum_{h_{a, b}=1}^{\bar{H}_{a, b}} \Delta_{n, i, j, a, b, h_{a, b}} \geq \sum_{k=1}^{L_{n, i}} \delta_{n, i, j, k}, \\
\forall n \in N, \forall i \in I_{n}, \forall j \in I_{n} .
\end{array}
$$

In Figure 2, for example, at intersection $n=13$, two paths exist for connecting two different origins and one destination. Both of them require a left-turn from $\operatorname{arm} i=1$ to $\operatorname{arm} j=2$. In this case, we have $\Delta_{n=13, i=1, j=2, a, b, h_{a, b}+1}=\Delta_{n=13, i=1, j=2, a^{\prime}, b, h_{a^{\prime}, b}}=1$ and therefore at least one left-turn lane marking on one of the approach lanes must exist; that is, $\sum_{k=1}^{L_{n, i}} \delta_{n=13, i=1, j=2, k} \geq 1$. Conversely, no path exists using straight-ahead movement and right-turn movement at intersection $n=13$, respectively, from arm $i=1$ to arm $j=3$ and from arm $i=1$ to arm $j=4$. Numerically, $\Delta_{n=13, i=1, j=3, a, b, h_{a, b}+1}=\Delta_{n=13, i=1, j=4, a, b, h_{a, b}+1}=$

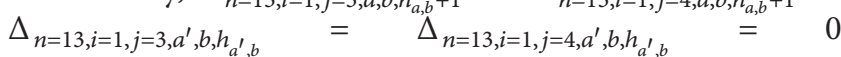
should be required, correspondingly. In turn, $M \sum_{a=1}^{A} \sum_{b=1}^{B} \sum_{h_{a, b}=1}^{\bar{H}_{a, b}} \Delta_{n=13, i=1, j=4, a, b, h_{a, b}}=0$ and it then forces $\sum_{k=1}^{L_{n, i}} \delta_{n=13, i=1, j=2, k}=0$. Lane markings for straightahead and right-turn movements are useless and should be prohibited for existence.

\section{Constraints for Individual Signalized Intersections}

3.1. Matching the Assigned Lane Flows with respect to the Demand Turning Flows at Intersections. From Section 2.3, (3) evaluates the turning flows $Q_{n, i, j}$ entering intersections $n$ from different $\operatorname{arm} i$ to different $\operatorname{arm} j$. These demand flows at an intersection will be further assigned onto different approach lanes $k$ according to the optimized lane marking patterns. A set of flow conservation constraints can be established as follows:

$$
Q_{n, i, j}=\sum_{k=1}^{L_{n, i}} q_{n, i, j, k}, \quad \forall i \neq j=1, \ldots, I_{n} ; \forall n=1, \ldots, N
$$

3.2. Establishing Minimum Lane Marking(s) on Approach Lanes. For practical designs, every approach traffic lane should be utilized to discharge traffic demand flows. At least one lane marking permitting a turn movement should be designed for each approach lane. Shared lane markings are also feasible if two or three movement turns are permitted on approach lanes at the same time:

$$
\begin{aligned}
\sum_{\forall j \neq i} \delta_{n, i, j, k} & \geq 1, \\
\forall k & =1, \ldots, L_{n, i} ; \forall i=1, \ldots, I_{n} ; \forall n=1, \ldots, N .
\end{aligned}
$$

3.3. Assigning Lane Markings without Exceeding Downstream Exit Lane Number. For each turn movement from arm $i$ to $\operatorname{arm} j$ at intersection $n$, number of exit lanes on arm $j$ should always be greater than or equal to the number of assigned lane markings to enter arm $j$. Undesirable traffic merging at downstream exit lanes may occur if number of lane markings is more than exit lane number. Constraint set in (13) should be given to control the number of lane markings:

$$
E_{n, j} \geq \sum_{k=1}^{L_{n, i}} \delta_{n, i, j, k}, \quad \forall i \neq j=1, \ldots, I_{n} ; \forall n=1, \ldots, N .
$$

3.4. Ensuring Consistency between Lane Markings and Assigned Lane Flows. If a traffic movement is prohibited on an approach traffic lane, the assigned lane flow of the 
associated turn movement should also be vanished. A linear constraint set can be defined as follows:

$$
\begin{aligned}
& M \delta_{n, i, j, k} \geq q_{n, i, j, k} \geq 0, \\
& \quad \forall k=1, \ldots, L_{n, i} ; \forall i \neq j=1, \ldots, I_{n} ; \forall n=1, \ldots, N,
\end{aligned}
$$

where $M$ is an arbitrary large positive number. If $\delta_{n, i, j, k}=0$, that is, the movement is prohibited, the respective lane flow $q_{n, i, j, k}$ must be zero. However, if $\delta_{n, i, j, k}=1$, that is, the turn movement is permitted, the assigned lane flow can take on any positive value, as long as it satisfies the demand flow relationship in (11).
3.5. Lane Markings across Adjacent Lanes. For any pair of adjacent approach traffic lanes, $k$ (on the left) and $k+1$ (on the right), from arm $i$, if a left-turn (or straight-ahead) movement to arm $j$ is permitted at lane $k+1$, then for safety reasons, the right-turn and straight-ahead (or right-turn) movement to other arms should all be prohibited along approach lane $k$, in order to eliminate any potential internal conflict within the same arm. A mathematical function $\Gamma(n, i, j)$ could be derived to identify a left-turn $\Gamma(n, i, j)=1$, a straight-ahead movement $\Gamma(n, i, j)=2$, or a right-turn $\Gamma(n, i, j)=3$. This can be specified by the following constraint set:

$$
\begin{aligned}
1-\delta_{n, i, j, k+1} \geq \delta_{n, i, j^{\prime}, k} \geq \delta_{n, i, j, k+1}-1 & \\
& \forall j^{\prime} \in\left[\Gamma(n, i, j)<\Gamma\left(n, i, j^{\prime}\right)\right] ; \forall i \neq j=1, \ldots, I_{n} ; \forall k=1, \ldots, L_{n, i}-1 ; n=1, \ldots, N .
\end{aligned}
$$

Given the binary nature of the variables, if $\delta_{n, i, j, k+1}=1$, then $\delta_{n, i, j^{\prime}, k}$ must vanish for all $j^{\prime} \in\left[\Gamma(n, i, j)<\Gamma\left(n, i, j^{\prime}\right)\right]$, that is, the turn movement $\left(n, i, j^{\prime}, k\right)$ is prohibited. On the other hand, if $\delta_{n, i, j, k+1}=0$, then the binary variable $\delta_{n, i, j^{\prime}, k}$ can take on any value of 0 or 1 freely.

3.6. Ranges of Operating Cycle Length. Define the minimum and maximum cycle lengths to be $c_{\min }$ and $c_{\max }$, respectively, for practical operations. A reciprocal of cycle length $\zeta=1 / c$ is modeled in the optimization process so as to eliminate the potential nonlinearity in signal timing variables. The feasible range of the operating cycle length can now be restrained by the following constraint set:

$$
\frac{1}{c_{\min }} \geq \zeta \geq \frac{1}{c_{\max }},
$$

which ensures that the operating cycle length will fall into the feasible range of $\left(c_{\min }, c_{\max }\right)$. Normally, $c_{\max }$ is 120.0 seconds in Hong Kong and $c_{\min }$ should be computed when all traffic signal settings are operating at minimum green duration times.

3.7. Synchronization of Traffic Signal Settings for Approach Lanes and Turns. For safety reasons, if an approach lane is designed with a shared lane marking, the involved movements should receive identical signal timings. From $\operatorname{arm} i$ at intersection $n$, if a turning movement to arm $j$ is permitted on lane $k$, the following constraint sets can be given to synchronize the traffic signal settings

$$
\begin{aligned}
& M\left(1-\delta_{n, i, j, k}\right) \geq \Theta_{n, i, k}-\theta_{n, i, j} \geq-M\left(1-\delta_{n, i, j, k}\right), \\
& M\left(1-\delta_{n, i, j, k}\right) \geq \Phi_{n, i, k}-\phi_{n, i, j} \geq-M\left(1-\delta_{n, i, j, k}\right),
\end{aligned}
$$

$\forall i \neq j=1, \ldots, I_{n} ; \forall k=1, \ldots, L_{n, i} ; \forall n=1, \ldots, N$, where $M$ is an arbitrary large positive number. If a movement $(n, i, j, k)$ is permitted along approach lane $k$ from $\operatorname{arm} i$ to $\operatorname{arm} j$ at intersection $n$, we have $\delta_{n, i, j, k}=1$ and hence the values on both sides of the inequalities in (17) become zero. This forces $\Theta_{n, i, k}=\theta_{n, i, j}$ and $\Phi_{n, i, k}=\phi_{n, i, j}$ from the constraint sets. Identical traffic signal settings for movement turns and approach lanes could be achieved. On the other hand, if this movement is not permitted, the constraint sets would be ineffective because $\left(1-\delta_{n, i, j, k}\right)$ is equal to " 1 " and hence different traffic signal settings could be operated.

3.8. Operating Ranges of Starts of Green Times. Since the traffic signal settings at intersections are cyclic in nature, green times can be started arbitrarily within a signal cycle as long as they satisfy other conditions and constraints in the problem formulation. Numerically, all starts of green times are confined within the range of $(0,1)$, that is, within one full traffic signal cycle

$$
1 \geq \theta_{n, i, j} \geq 0, \quad \forall i \neq j=1, \ldots, I_{n} ; n=1, \ldots, N
$$

\subsection{Operating Upper and Lower Bounds of Duration of Green} Times. Duration of green times operating for an approach traffic lane is subject to a minimum value, called minimum green times. Since all traffic signal timings are operating in a signal cycle, the longest green duration times should be one full signal cycle. These upper and lower bounds can be restricted as follows:

$$
1 \geq \phi_{n, i, j} \geq g_{n, i, j} \zeta, \quad \forall i \neq j=1, \ldots, I_{n} ; n=1, \ldots, N
$$

3.10. Regulating Displaying Sequence of Traffic Signal Timings. Any two signal groups (or movements), $(n, i, j)$ and $\left(n, i^{\prime}, j^{\prime}\right)$, are said to be incompatible within an intersection $n$, if movement paths $(n, i, j)$ and $\left(n, i^{\prime}, j^{\prime}\right)$ have intersection point at the common area of an intersection. Denote the set of incompatible signal groups as $\bar{\Psi}_{n}$, which can be derived from $\Psi_{n}$, the set of incompatible movements. For any two incompatible signal groups $(n, i, j)$ and $\left(n, i^{\prime}, j^{\prime}\right)$ in $\Psi_{n}$, the order of signal displays is governed by a successor function [22], $\Omega_{n, i, j, i^{\prime}, j^{\prime}}$, where $\Omega_{n, i, j, i^{\prime}, j^{\prime}}=0$ if the start of green of signal 
group $\left(n, i^{\prime}, j^{\prime}\right)$ follows that of signal group $(n, i, j)$; and $=1$ if the opposite is true. Therefore, the following constraints can be set for the successor functions:

$$
\begin{aligned}
& \Omega_{n, i, j, i^{\prime}, j^{\prime}}+\Omega_{n, i^{\prime}, j^{\prime}, i, j}=1, \\
& \forall\left((n, i, j),\left(n, i^{\prime}, j^{\prime}\right)\right) \in \bar{\Psi}_{n} .
\end{aligned}
$$

3.11. Provision of Clearance Times Separating Conflicting Movements. For any pair of incompatible movements, clearance time constraints are required when both movements are permitted through establishing the respective lane markings. For two incompatible traffic movements defined as $u_{n}=$ $(n, i, j, k)$ and $v_{n}=\left(n, i^{\prime}, j^{\prime}, k^{\prime}\right)$, the following constraint set can be given to satisfy the clearance time requirements:

$$
\begin{gathered}
\theta_{n, i^{\prime}, j^{\prime}}+\Omega_{n, i, j, i^{\prime}, j^{\prime}}+M\left(2-\delta_{n, i, j, k}-\delta_{n, i^{\prime}, j^{\prime}, k^{\prime}}\right) \\
\geq \theta_{n, i, j}+\phi_{n, i, j}+\omega_{n, u_{n}, v_{n}} \zeta, \quad \forall\left(u_{n}, v_{n}\right) \in \Psi_{n},
\end{gathered}
$$

where $M$ is an arbitrary large positive number. The constraint set is effective only when the two incompatible movements are permitted and the two respective lane markings exist; that is, $\delta_{n, i, j, k}=\delta_{n, i^{\prime}, j^{\prime}, k^{\prime}}=1$.

3.12. Identical Flow Factors across Adjacent Approach Lanes with Identical Lane Marking. Assigning demand traffic flows to approach lanes is based on the queuing theory, for which the degrees of saturation on a pair of adjacent lanes, with at least one common lane marking, must be identical. From Section 3.7, the signal settings on this pair of adjacent lanes must be identical. To ensure identical degrees of saturation, the flow factors (defined as the total assigned flow divided by the saturation flow) of these adjacent lanes should be equalized. Let $y_{n, i, k}$ be the flow factor on approach lane $k$ from $\operatorname{arm} i$ at intersection $n$, which can be expressed as

$$
\begin{aligned}
y_{n, i, k}= & \frac{\sum_{\forall j \neq i} q_{n, i, j, k}}{s_{n, i, k}}, \\
& \forall k=1, \ldots, L_{n, i} ; \forall i=1, \ldots, I_{n} ; \forall n=1, \ldots, N .
\end{aligned}
$$

Since

$$
f_{n, i, j, k}=\frac{\sum_{j \neq i} q_{n, i, j, k}-q_{n, i, j^{\prime}, k}}{\sum_{j \neq i} q_{n, i, j, k}}, \quad \forall j^{\prime} \in[\Gamma(n, i, j)=2] ; \forall k=1, \ldots, L_{n, i} ; \forall i=1, \ldots, I_{n} ; \forall n=1, \ldots, N,
$$

where $\Gamma(n, i, j)=2$ is a straight-ahead movement, we can show using the saturation flow prediction equation in [23]

$$
\begin{aligned}
y_{n, i, k}= & \frac{1}{\bar{s}_{n, i, k}} \sum_{j \neq i}\left(1+\frac{1.5}{r_{n, i, j, k}}\right) q_{n, i, j, k}, \\
& \forall k=1, \ldots, L_{n, i} ; \forall i=1, \ldots, I_{n} ; \forall n=1, \ldots, N .
\end{aligned}
$$

To equalize flow factors for two adjacent approach lanes with identical lane marking(s), we have

$$
\begin{aligned}
M( & \left.-\delta_{n, i, j, k}-\delta_{n, i, j, k+1}\right) \\
\geq & \frac{1}{\bar{s}_{n, i, k}} \sum_{j \neq i}\left(1+\frac{1.5}{r_{n, i, j, k}}\right) q_{n, i, j, k} \\
& \quad-\frac{1}{\bar{s}_{n, i, k+1}} \sum_{j \neq i}\left(1+\frac{1.5}{r_{n, i, j, k+1}}\right) q_{n, i, j, k+1} \\
\geq & -M\left(2-\delta_{n, i, j, k}-\delta_{n, i, j, k+1}\right), \\
\forall k & =1, \ldots, L_{n, i}-1 ; \forall i=1, \ldots, I_{n} ; \forall n=1, \ldots, N,
\end{aligned}
$$

where $M$ is an arbitrary large positive number and $k$ (on the left) and $k+1$ (on the right) are two adjacent approach lanes from $\operatorname{arm} i$ at intersection $n$. The constraint sets are effective only when $\delta_{n, i, j, k}=\delta_{n, i, j, k+1}=1$.

3.13. Maximum Acceptable Degree of Saturation. Let $p_{n, i, k}$ be the maximum degree of saturation on approach lane $k$ from $\operatorname{arm} i$ at the intersection $n$ in a study signal-controlled network. For an approach traffic lane $k$ from arm $i$ at intersection $n$, the degree of saturation can be given by

$$
\begin{aligned}
\rho_{n, i, k}= & \frac{y_{n, i, k}}{\Phi_{n, i, k}+e \zeta}, \\
& \forall k=1, \ldots, L_{n, i} ; i=1, \ldots, I_{n} ; n=1, \ldots, N,
\end{aligned}
$$

where $\rho_{n, i, k}$ and $e$ are the degree of saturation on lane $k$ from arm $i$ at intersection $n$ and the difference between actual and effective greens (measured in time unit, usually taken as 1 second), respectively. The following constraint set can be given to ensure that the degree of saturation is below the maximum acceptable limit:

$$
\begin{aligned}
\Phi_{n, i, k}+e \zeta \geq \frac{1}{p_{n, i, k} \bar{s}_{n, i, k}} \sum_{j \neq i}\left(1+\frac{1.5}{r_{n, i, j, k}}\right) q_{n, i, j, k}, \\
\quad \forall k=1, \ldots, L_{n, i} ; \forall i=1, \ldots, I_{n} ; \forall n=1, \ldots, N .
\end{aligned}
$$

\section{Objective Function for Optimization}

Usually, there are three standard objectives for optimizing traffic signal settings: (1) capacity maximization, (2) cycle length minimization, and (3) delay minimization for individual signalized intersections. In the present study, traffic signal settings are optimized based on criteria (1) because they can be effectively formulated as Binary-Mixed-Integer-LinearProgramming (BMILP) problems and standard branch-andbound routines could be applied to solve the optimum 
TABLE 1: Demand OD flows $T_{a, b}$.

\begin{tabular}{lcccccc}
\hline & \multicolumn{1}{c}{ Destination, $b$} & \multicolumn{2}{c}{ Total flows from origin } \\
& \multirow{2}{*}{$T_{a, b}(\mathrm{pcu} / \mathrm{h})$} & 1 & 2 & 3 & 4 & 1,000 \\
Origin, $a$ & 1 & - & 200 & 300 & 500 & 1,100 \\
& 2 & 300 & - & 200 & 600 & 500 \\
\hline \multicolumn{2}{l}{ Total flows entering destination } & 100 & 200 & - & 200 & 500 \\
\hline
\end{tabular}

solution [19]. The problem of delay minimization involves nonlinear objective mathematical function which is a highly nonlinear and nonconvex mixed-integer problem in the lanebased optimization framework [17]. We may leave this hard problem in future study.

For capacity maximization, we will borrow the concept that has been widely adopted for individual intersections. We introduce the common flow multiplier, $\mu$ in which the demand turning flow matrix (demand flows from different arms to different arms) is multiplied by the single common flow multiplier $\mu[19,21]$. The maximization process is to keep increasing the common flow multiplier and in turn enlarging the demand flow inputs to a signal-controlled system until the system reaches the maximum degree of saturation. Similarly, in the proposed signal-controlled network system, we take on the OD demand flows (matrix) as inputs. The scaled OD demand flows by the single common flow multiplier will be entered to the modeling network system until the maximum degree of saturation is reached for all the critical approach lanes serving for different turn movements matching the optimized set of lane markings. The resultant lane markings at various intersections and approach lanes should be linked up for connections of all network links. The assigned lane flows according to the optimized set of lane markings could be combined to form the respective path flows. And all path flows should be able to match the given OD demand flow patterns as the flow conservation purposes. The capacity maximization problem can therefore be formulated as a Binary-Mixed-Integer-Linear-Program:

$$
\underset{\Lambda=\left(\Lambda_{b}, \Lambda_{c}\right)}{\operatorname{maximize}} \mu
$$

subject to constraints in (1)-(27). Standard branch-andbound solution technique could be applied to solve the optimum solutions (refer to the Appendix for the general solution process).

\section{Case Study}

In this section, we would like to demonstrate the proposed formulation to design a network configuration and optimize the link connectivity. A 4-intersection network is modeled, $n=4$. Figure 3 shows the general arrangement of the case study network. There are 2 approach lanes, $L_{n, i}=2$, and 2 exit lanes, $E_{n, j}=2$, for all arms at all intersections. All left-hand lane is nearside lane and right-hand lane is nonnearside lane with saturation flows of $\bar{s}_{n, i, k=1}=1,965$ and $\bar{s}_{n, i, k=2}=2,080 \mathrm{pcu} / \mathrm{h}$ (for straight-ahead movements), respectively. Lane markings (turning arrows) are free to design on approach traffic lanes. Saturation flows will be revised according to the turning proportions obtained from the optimized lane flow results. 4 nodes are modeled as origins and destinations. Users' input demand flows are tabulated in Table 1 . For example, arm $i=1$ at intersection $n=1$ is the origin node $a=1$ and destination node $b=1$. From the input demand flows in Table 1, all demand flows from origins to destinations are given.

Visually, we could expect that demand flows from one origin may have two feasible paths to reach other destinations. For example, from origin $a=1$ to destination $b=3$, the demand flow input is $300 \mathrm{pcu} / \mathrm{h}$ and these flows could enter intersections $n=1$ and then $n=2$ and then $n=3$ or enter intersections $n=1$ and then $n=4$ and then $n=3$ to reach the destination depending on the provisions of lane markings. If all relevant lane markings are available depending on the optimization results, then one of the two paths or both paths could be existed to carry the demand flow inputs. In the case study, Figure 4 plots all the feasible paths that could exist to connect all OD pairs. It is worth noting that, for the OD pair from origin $a=1$ to destination $b=2$, the first path $h_{1,2}=1$ is a shorter path that only passes through two intersections to reach the destination while the second path $h_{1,2}=2$ is a longer path that needs to pass through four intersections. Similar short and long paths are found in some other OD pairs such as origin $a=2$ and destination $b=1$. To effectively demonstrate our proposed design method to optimize the lane marking patterns at intersections to connect all the given OD pairs, we allow three OD pairs $(a=1, b=3),(a=1, b=$ $4)$, and ( $a=2, b=4)$ with path choices and all remaining OD pairs are restricted to use the shorter path only (passing through less numbers of intersections). It would be clearer to illustrate the optimization results. Paths in solid lines in Figure 4 will be modeled and other paths in dashed lines are disabled in the case study. Turning radius is set to be $12.0 \mathrm{~m}$ for all left- and right-turn movements from both nearside and nonnearside lanes. All $\sigma_{n, a, b, h_{a, b}}$ are all set to be 1.0 assuming all input demand flows once entering the network system will appear at all downstream intersections. The arbitrary large positive number will take on $M=10,000$. Clearance times and minimum green duration times are 6.0 and 5.0 seconds, respectively. Effective green duration times are 1.0 second longer than the actual (or displayed) green duration times; that is, $e=1.0$ second. Maximum degree of saturation for all approach lanes is set to be 0.9 .

Based on all the above users' inputs, then the proposed formulation can be applied to optimize the common flow 


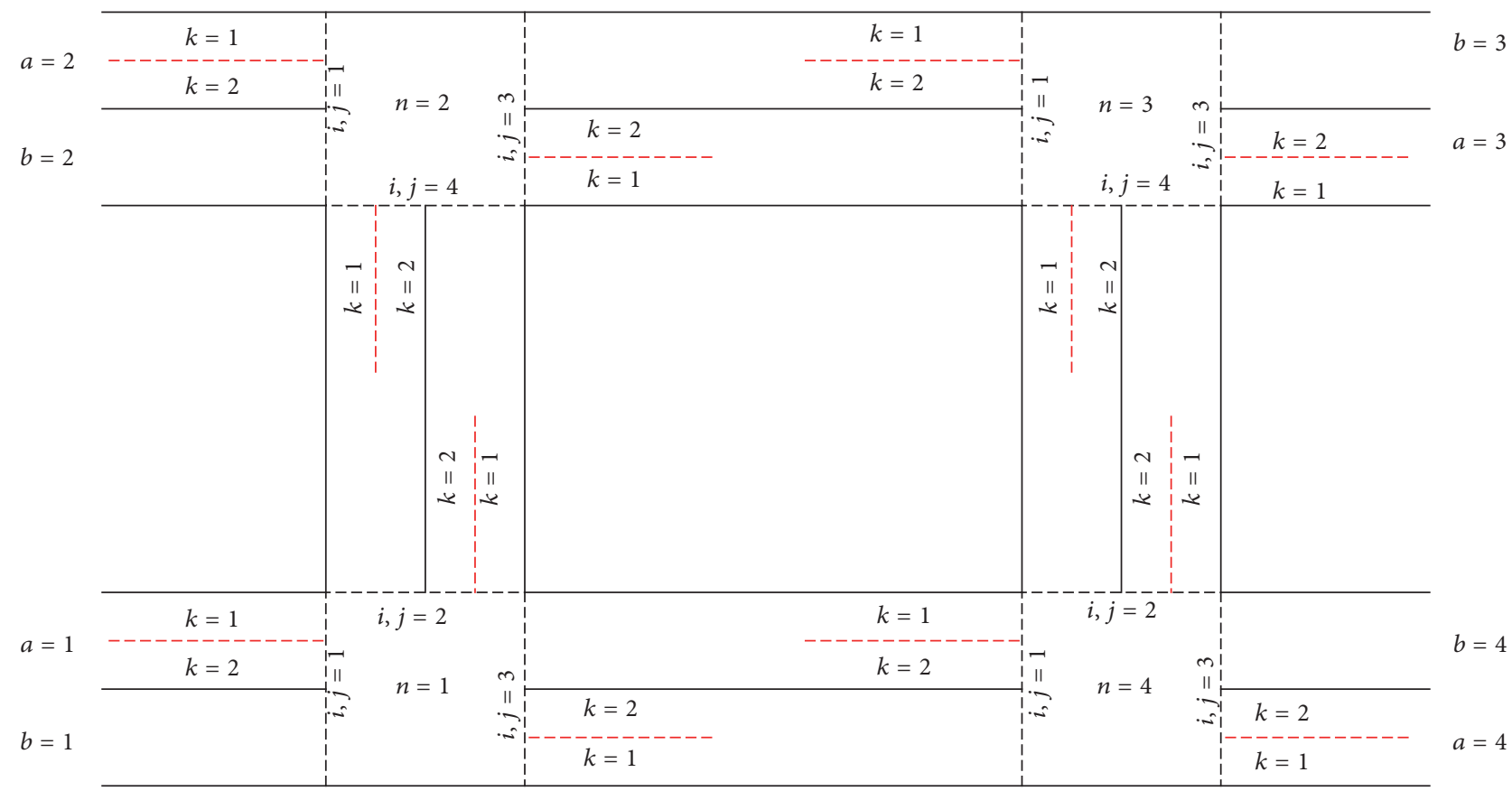

$\begin{array}{ll}a \text { : origin } & i \text { : from arm } \\ b \text { : destination } & j: \text { to arm } \\ n \text { : intersection } & k \text { : lane }\end{array}$

FIGURE 3: A case study signal-controlled network with 4 signalized intersections.

Origin $a$

Destination $b$

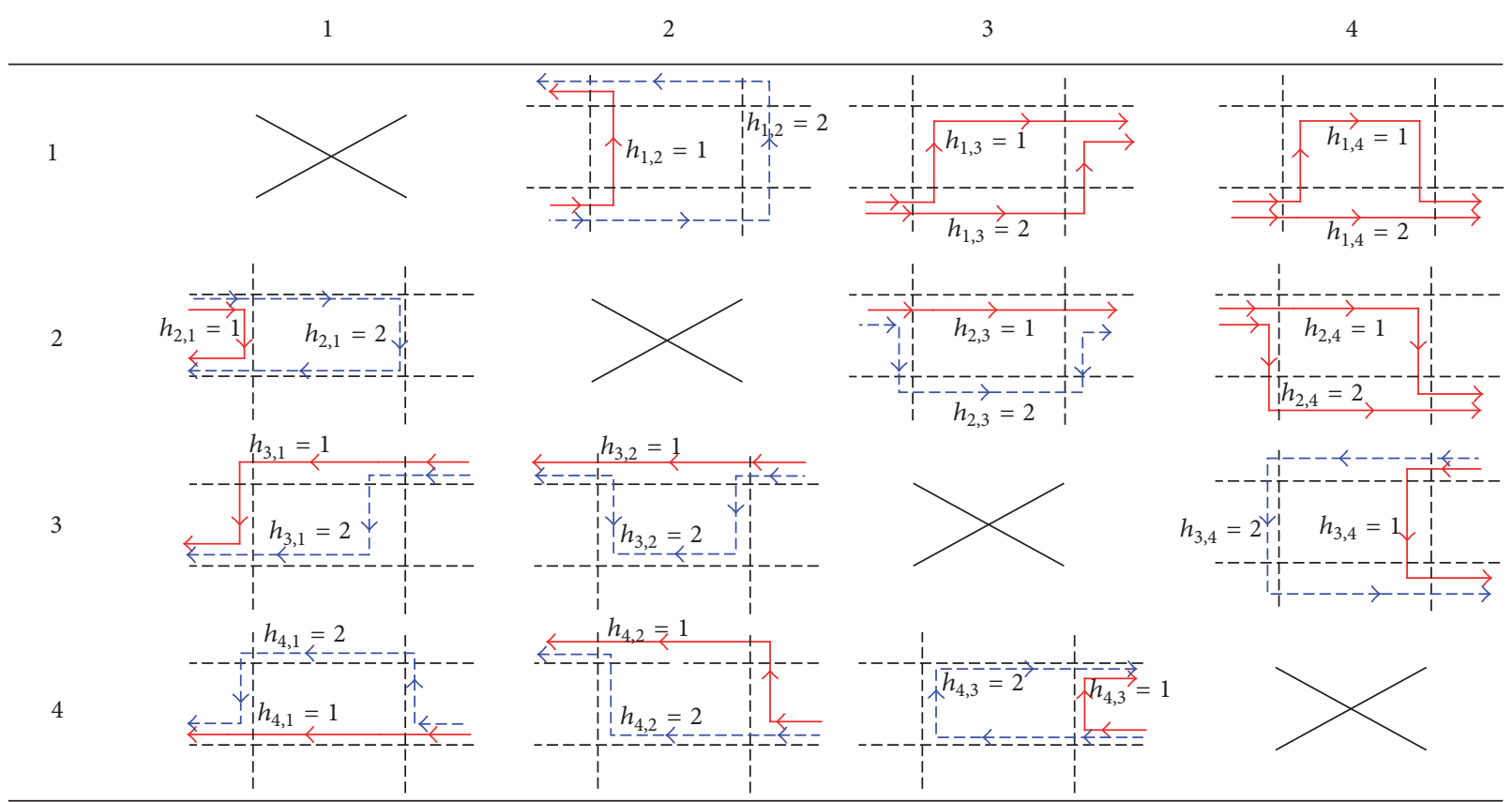

FIGURE 4: Feasible paths in the case study network connecting the 4 OD pairs. 


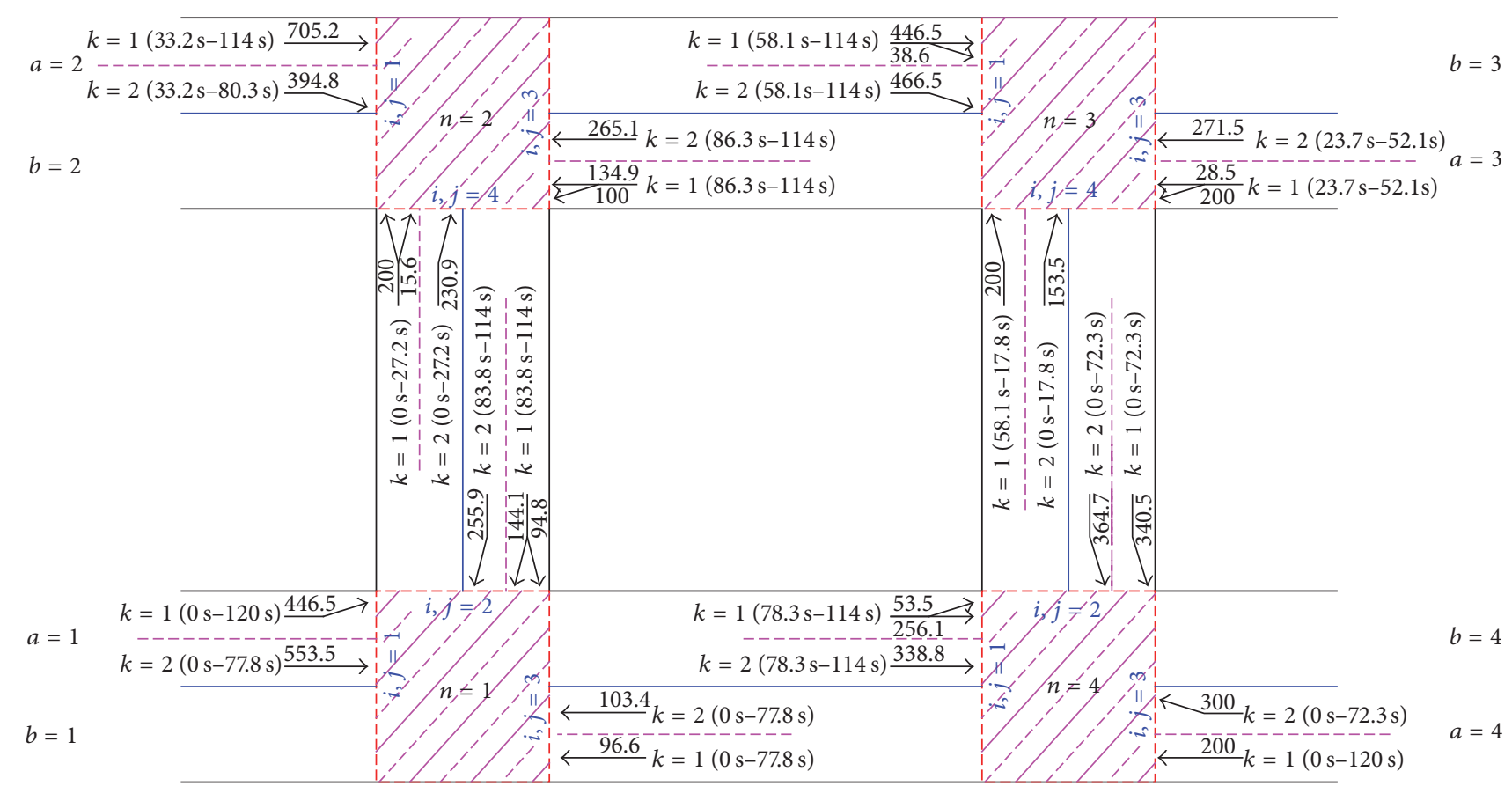

FIGURE 5: Optimized network configurations including lane markings and assigned lane (turning) flows at individual intersections.

multiplier to maximize the capacity of the signal-controlled network. And the optimized common flow multiplier $\mu=$ 1.7106 with $71.06 \%$ reserve capacity (for the same sets of demand flow inputs and problem settings, if the signalcontrolled network is operated with a set of worse noncooperated lane markings, the common flow multiplier can be as low as 0.8517 implying that the system is overloaded by $14.83 \%$ ). Figure 5 gives detailed optimization results on lane markings, assigned lane flows, and traffic signal settings. For example, at intersection $n=1$, from arm $i=1$, a left-turn lane marking is optimized to allow left-turn traffic to enter arm $j=2$ along the nearside lane $k=1$. Next to the nearside lane, there is another nonnearside lane $k=2$ with a straight-ahead lane marking for traffic turning from arm $i=1$ to arm $j=3$. Starts of green times and ends of green times (in seconds) are given inside brackets. Their differences (ends of green times - starts of green times) are the actual green duration times. All effective green times equal actual green times plus one second numerically due to $e=1.0$ second. Numbers next to the lane marking arrows are the respective assigned lane turning flows in $\mathrm{pcu} / \mathrm{h}$. Inside the 4 -intersection network, 6 shared lane markings are optimized to permit more than one turning movement along one approach lane. For each of the four intersections in the case study intersection, it consists of 3 arms (T-intersection) and there are two approach lanes and two exit lanes in each arm. Details of assigned lane flows which should be consistent with the lane markings are given in Tables 2-5. Column 1 is the origin arm of the turning flows. Column 2 is the approach lane number. Columns 3-5 give the individual assigned lane flows. Column 6 is the total lane flows that are the sum of all assigned turning flows. Column 7 is the turning proportion along the approach lanes which will be used to revise the lane saturation flows. Column 8 tabulates the revised lane saturation flows taking the lane turning proportion into account. Column 9 (=Column 6/Column 8) is the flow factor for each individual approach lane. Columns 10 and 11 are the starts of green times and effective green duration times. The last Column 12 is the degree of saturation for approach lane. To verify the proposed formulation is robust, it is necessary to examine whether the assigned lane flows can be matching with the users' given demand flow patterns in Table 1. Referring to the remarks under Tables 2-5, the total assigned lane flows from corresponding arms (and approach lanes) can be retrieved and their summation flow values can be evaluated to exactly match the given demand flows. From Table 2, for example, arm $i=1$ at intersection $n=1$ is the origin $a=1$; therefore the row sum of the total flow from arm $i=1$ is $1,000 \mathrm{pcu} / \mathrm{h}$ which is the same as the total flow from origin $a=1$ given in Table 1 (the last column). Similarly, demand flows from other three origins and exit flows entering the four destinations can all be traced similarly according to the remarks under Tables 2-5.

Another set of outputs that is worth discussing is the path flows. We mentioned earlier that only three OD pairs $(a=1, b=3),(a=1, b=4)$, and $(a=2, b=4)$ are given path choices. And all other OD pairs are restricted to use their shorter paths. Figure 6 presents the optimized path flow details. All paths given in solid lines in Figure 4 are all considered in the optimization process. For OD pair ( $a=1, b=4)$, we provide path choices for selections. Indeed, path $h_{a, b}=1$ is a longer path passing through four intersections and $h_{a, b}=2$ is a shorter one and thus the optimization results for these two paths are $P_{a=1, b=4, h_{a, b}=1}=$ $0.00 \mathrm{pcu} / \mathrm{h}$ and $P_{a=1, b=4, h_{a, b}=2}=500.00 \mathrm{pcu} / \mathrm{h}$, respectively. 


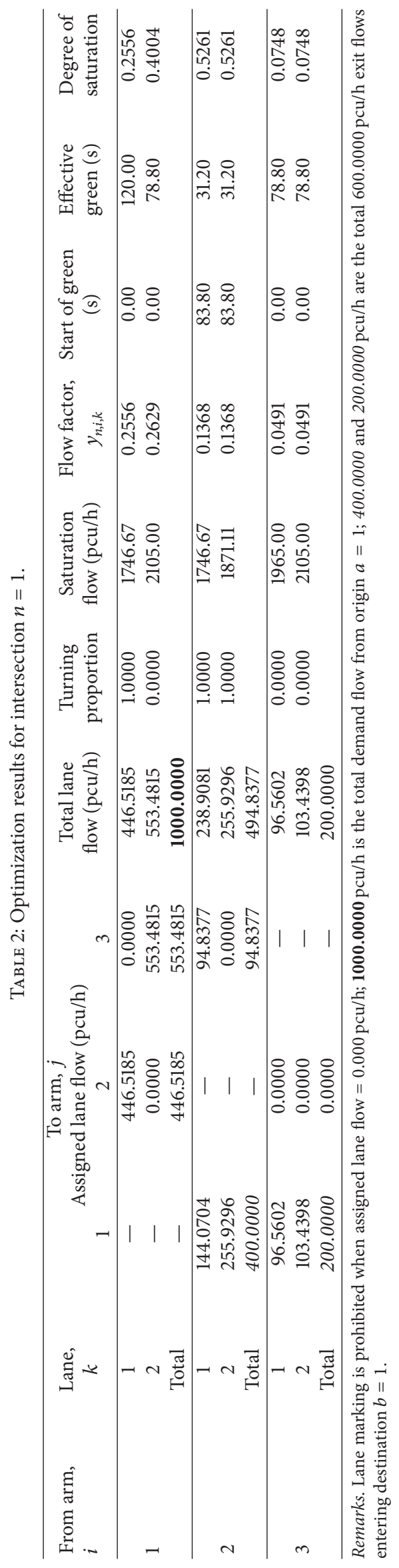




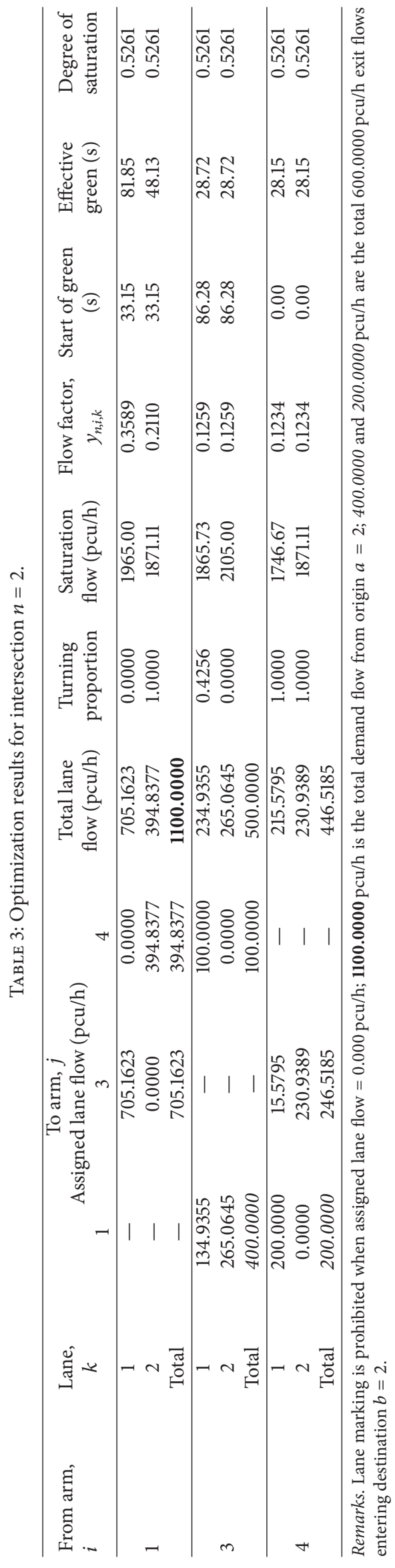




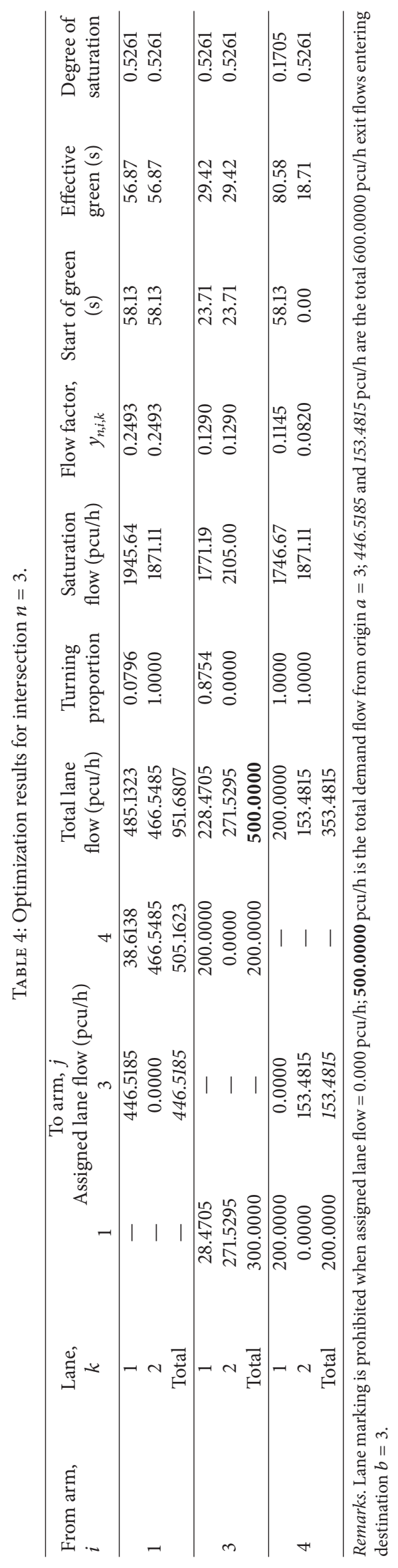




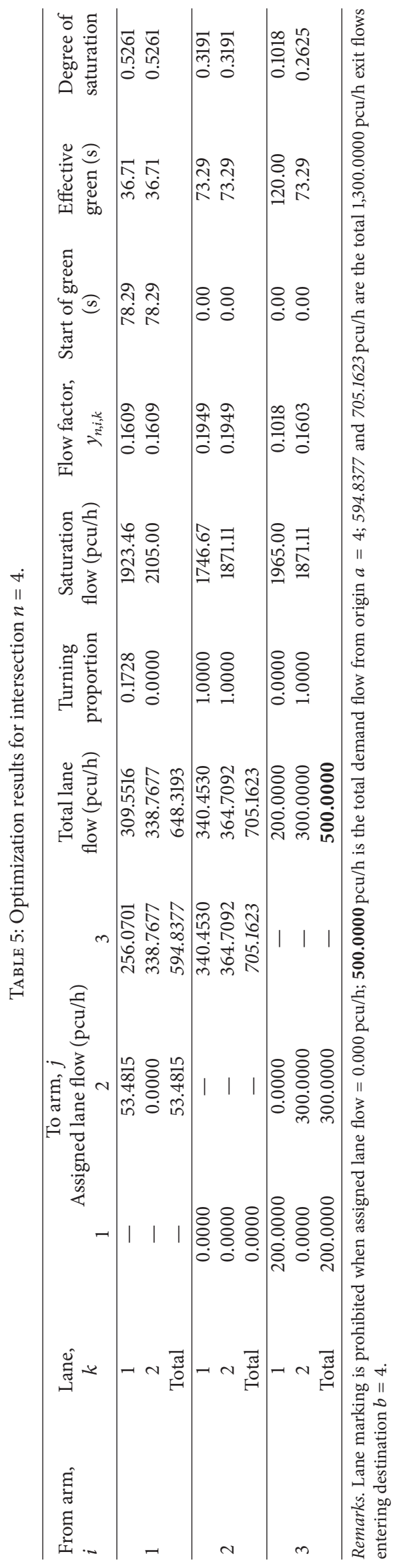




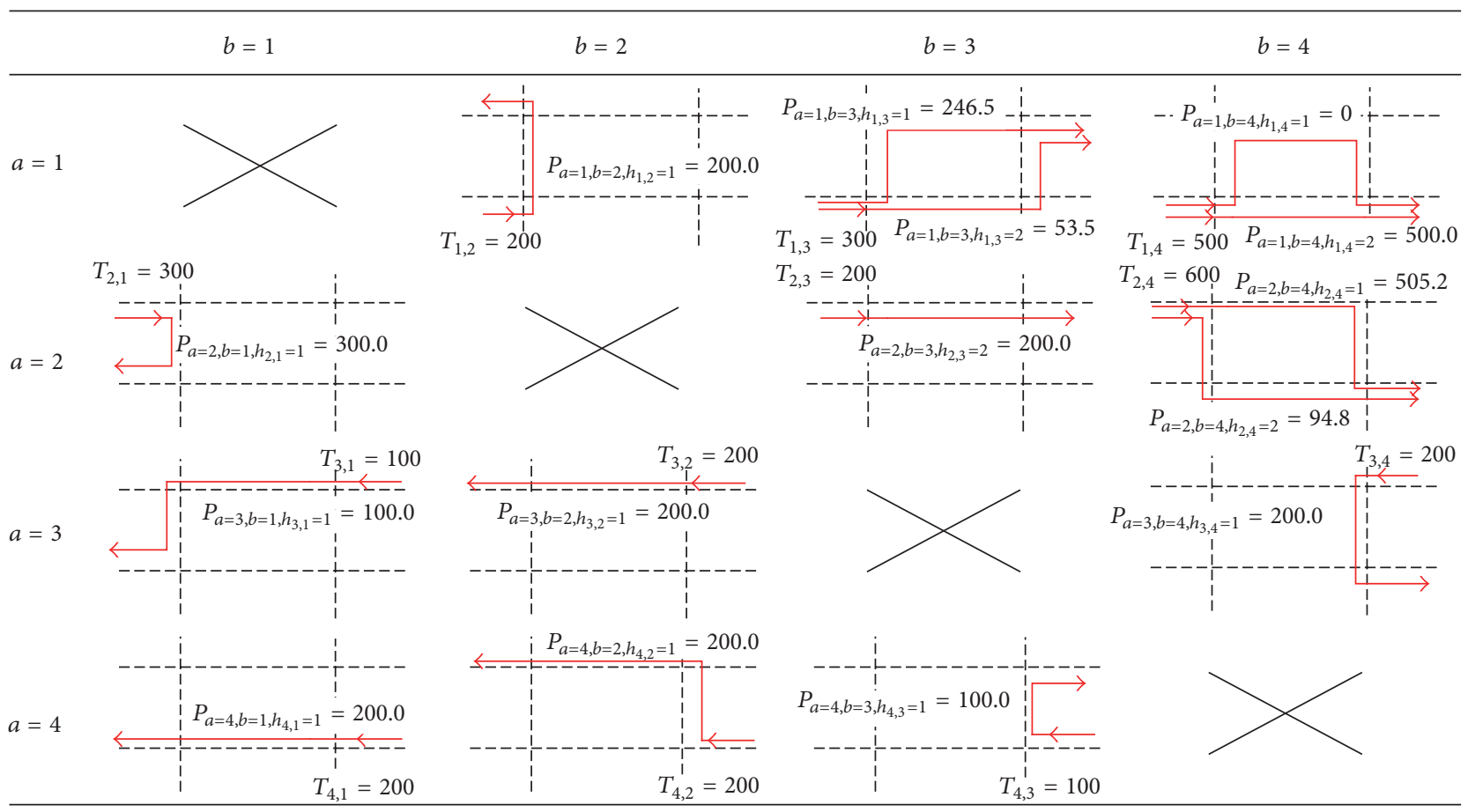

FIGURE 6: Optimized path flow details for the case study network matching the given OD demand flows (in pcu/h).

It shows logical result. As for the OD pair $(a=1, b=$ 3 ), the users' input demand flow is $T_{a=1, b=3}=300 \mathrm{pcu} / \mathrm{h}$ and the two path flows are $P_{a=1, b=3, h_{a, b}=1}=246.5 \mathrm{pcu} / \mathrm{h}$ and $P_{a=1, b=3, h_{a, b}=1}=53.5 \mathrm{pcu} / \mathrm{h}$. And for the OD pair $(a=2, b=$ 4), the users' input demand flow is $T_{a=2, b=4}=600 \mathrm{pcu} / \mathrm{h}$ and the two path flows are $P_{a=2, b=4, h_{a, b}=1}=505.2 \mathrm{pcu} / \mathrm{h}$ and $P_{a=2, b=4, h_{a, b}=2}=94.8 \mathrm{pcu} / \mathrm{h}$. For other OD pairs, all demand flows will be assigned onto the available paths. All these path flows will then serve as demand turning flow inputs at individual intersections for optimizing the lane markings and traffic signal settings at intersection levels as shown in Tables $2-5$.

Overall, to model the case study network containing four signalized intersections with two approach lanes and two exit lanes for each arm, there are totally 617 variables including 405 binary-type integer variables and 212 continuous variables. Number of linear governing constraints is 1,955 for binding the 617 variables. It takes less than 5 minutes of computing time in an intel i7 CPU of $2.67 \mathrm{GHz}$ with $8.0 \mathrm{~GB}$ memory (RAM) to reach the global optimum solution.

\section{Conclusions}

In the proposed study, we successfully extend the lane-based design concept to build a new mathematical optimization model to optimize the individual lane usage including the lane markings, assigned lane flows, and their corresponding traffic signal settings. The optimized design provides multiple paths to connect the given origin and destination pairs through different turns at different intersections according to the optimized lane markings. Link connectivity and network configuration are enhanced and optimized in a unified framework together with the assigned lane flows and traffic signal settings. It has been found that the performance of the case study network would be overloaded by $14.83 \%$ when a set of noncooperated lane markings (without shared lane marking design) is implemented. Using the proposed optimization method, the entire signal-controlled network could be improved by possessing $+71.06 \%$ reserve capacity (instead of being overloaded). The main contribution of this optimization result is to fix the lane markings to build a signal-controlled network for further sophisticated network analysis. The problem is formulated as a Binary-MixedInteger-Linear-Program (BMILP) which has been solved by a standard branch-and-bound routine for the global optimum solution. In the example, 4-intersection network with two approach and exit lanes, 405 binary variables, 212 continuous variables, and 1,955 linear governing constraints are required. Computation time is less than 5 minutes using a Gurobi solver in an i7 CPU computer system. We conclude that the proposed method is efficient and effective to design the signal-controlled network problem.

The proposed method does not take into account users' responses to the optimized network configuration settings. The modeling results serve like a system optimal setting in which the signalized intersections in a network could be optimized if users from origins could straightly follow the lane markings making their turns at intersections to reach their destinations ideally. Actual network performance could be deviated under users' equilibrium patterns. For further investigations, nonlinear link travel times and delays at intersections should be modeled so that proper responses 
from users and users' equilibrium patterns for realistic route choices could be obtained for better evaluations of the optimized network configurations.

\section{Appendix}

The BMILP problems for the capacity maximization can be standardized into the integer programming problem defined as follows:

$$
\begin{array}{ll}
\operatorname{maximize} & \mathbf{c}^{T} \mathbf{x} \\
\text { subject to } & \mathbf{A x} \leq \mathbf{b}, \\
& \mathbf{x} \geq 0,
\end{array}
$$

where $\mathbf{x}$ is a vector collecting all model variables including continuous and integer components and $\mathbf{c}$ is a vector of coefficients multiplied by the vector of model variables to form the objective function. $\mathbf{A}$ and $\mathbf{b}$ are coefficient matrix and a constant vector for setting up the problem constraints, respectively. A standard branch-and-bound technique starts by solving the program as if all integer variables are not restricted to being integers. It turns the original problem into a general linear programming (LP). The optimal solution can be found easily by either the simplex method or other graphical methods. The solution process can stop here if all the integer variables are found to be integers in this relaxed LP problem. The other strategy is to round each noninteger variable solution obtained from the LP results to its nearest integer as the optimal solution for the original integer program. However, some of the constraints may be violated during this rounding process making the solution infeasible. Thus, the problem has to be branched into two subproblems in which the solution space is divided into two regions. It requires a branching variable, which is normally picked from one of the integer variables, whose numerical value is less near to an integer. Based on the solution found in the LP problem relaxed from the original integer program, the two nearest integers of the branching variable solution become the two new bounds, which are then added to the two subproblems, in the form of constraints. For instance, one of the integer variables, $x_{0}$, is set as the branching variable and has a numerical value of 1.45 from the LP problem. Since $x_{0}$ is supposed to be an integer, then the two new bounds can be set as $x_{0} \leq 1$ and $x_{0} \geq 2(=0$ or $=1$ for binary variables) and added to the original constraint set to form the two subproblems without removing any feasible solution space. In each subproblem, if not all the integer variables have integer solutions, then further branching is required, generating a tree of subproblems. Once a subproblem is infeasible, then no further branching is necessary along that subproblem. An optimal solution found in a subproblem though it is feasible in the full problem, is not necessarily globally optimal. However, it can be regarded as a criterion to compare and trim the rest of the tree. If the objective solutions of those subproblems waiting for further branching (i.e., infeasible solutions because not all integer variables have integer solutions) are not better than the one from a feasible subproblem (i.e., a feasible solution because all integer variables have integer solutions), then further branching for those infeasible subproblems is not necessary and should be removed from further consideration. The search continues until all branching subproblems have been solved or no further branching subproblems exist. A computer package, called the MPL modeling system, integrating a Gurobi or CPLEX solver is used to implement the branchand-bound algorithm to solve the present BMILP for the

\begin{tabular}{|c|c|}
\hline$n, m:$ & Intersection number \\
\hline$N:$ & $\begin{array}{l}\text { Total number of intersections in a } \\
\text { signal-controlled network }\end{array}$ \\
\hline$i, j:$ & Arm number of an intersection \\
\hline$I_{n}:$ & Total number of arms at intersection $n$ \\
\hline$k:$ & Approach lane number \\
\hline$L_{n, i}:$ & $\begin{array}{l}\text { Total number of approach lanes from arm } \\
i \text { at intersection } n\end{array}$ \\
\hline$E_{n, j}:$ & $\begin{array}{l}\text { Number of exit lanes on arm } j \text { at } \\
\text { intersection } n\end{array}$ \\
\hline$a:$ & $\begin{array}{l}\text { Origin node in a study signal-controlled } \\
\text { network }\end{array}$ \\
\hline$b:$ & $\begin{array}{l}\text { Destination node in a study } \\
\text { signal-controlled network }\end{array}$ \\
\hline$A:$ & $\begin{array}{l}\text { Total number of origin nodes in a study } \\
\text { signal-controlled network }\end{array}$ \\
\hline$B:$ & $\begin{array}{l}\text { Total number of destination nodes in a } \\
\text { study signal-controlled network }\end{array}$ \\
\hline$h_{a, b}:$ & $\begin{array}{l}\text { Path number from origin } a \text { to destination } \\
b\end{array}$ \\
\hline $\bar{H}_{a, b}:$ & $\begin{array}{l}\text { Total number of paths connecting origin } a \\
\text { and destination } b\end{array}$ \\
\hline$H_{a, b}:$ & $\begin{array}{l}\text { Set of paths connecting origin } a \text { and } \\
\text { destination } b\end{array}$ \\
\hline$H_{a, b}^{\prime}$ & $\begin{array}{l}\text { A subset extracted from } H_{a, b}\left(H_{a, b}^{\prime} \subset H_{a, b}\right) \\
\text { in which the same lane marking from arm } \\
i \text { to arm } j \text { at intersection } n \text { will be used to } \\
\text { connect origin } a \text { and destination } b\end{array}$ \\
\hline$F(n, i, j):$ & $\begin{array}{l}\text { A mathematical function to identify origin } \\
a \text { and destination } b \text { along path } h_{a, b} \text { that } \\
\text { will make a turn from arm } i \text { to } \operatorname{arm} j \text { at } \\
\text { intersection } n\end{array}$ \\
\hline$\left.h_{a, b}\right):$ & $\begin{array}{l}\text { A mathematical function to identify all the } \\
\text { turns from arm } i \text { to arm } j \text { on lane } k \text { at } \\
\text { intersection } n \text { for a path } h_{a, b} \text { connecting } \\
\text { origin } a \text { and destination } b\end{array}$ \\
\hline$\Gamma(n, i, j):$ & $\begin{array}{l}\text { A mathematical function to identify the } \\
\text { turn direction (s) from arm } i \text { to arm } j \text { at } \\
\text { intersection } n \text { in which } \Gamma(n, i, j)=1 \text { is a } \\
\text { left-turn, } \Gamma(n, i, j)=2 \text { is a straight-ahead } \\
\text { movement, or } \Gamma(n, i, j)=3 \text { is a right-turn }\end{array}$ \\
\hline M: & An arbitrary large positive number \\
\hline $\bar{\Psi}_{n}$ & $\begin{array}{l}\text { Set of incompatible signal groups at } \\
\text { intersection } n\end{array}$ \\
\hline$\Psi_{n}:$ & $\begin{array}{l}\text { Set of incompatible turn movements at } \\
\text { intersection } n\end{array}$ \\
\hline
\end{tabular}
capacity maximization problem.

\section{Notations}




\begin{tabular}{|c|c|}
\hline$T_{a, b}:$ & $\begin{array}{l}\text { Demand flow inputs from origin } a \text { to } \\
\text { destination } b\end{array}$ \\
\hline$\delta_{n, i, j, k}:$ & $\begin{array}{l}\text { Permitted movement (lane marking) from } \\
\operatorname{arm} i \text { to arm } j \text { on lane } k \text { at intersection } n(=1 \\
\text { if it exists or }=0 \text { if it does not exist) }\end{array}$ \\
\hline$\Omega_{n, i, j, i^{\prime}, j^{\prime}}:$ & $\begin{array}{l}\text { Successor functions for any two } \\
\text { incompatible signal groups }(n, i, j) \text { and } \\
\left(n, i^{\prime}, j^{\prime}\right) \text { at intersection } n\end{array}$ \\
\hline$\alpha_{a, b, h_{a, b}}:$ & $\begin{array}{l}\text { Existence of a path } h_{a, b} \text { connecting origin } a \\
\text { and destination } b\end{array}$ \\
\hline$\beta_{a, b}:$ & $\begin{array}{l}\text { Existence of demand flow from origin } a \text { to } \\
\text { destination } b\end{array}$ \\
\hline$\Delta_{n, i, j, a, b, h_{a, b}}:$ & $\begin{array}{l}\text { An auxiliary binary variable to represent the } \\
\text { existence of a lane marking permitting a turn } \\
\text { from arm } i \text { to arm } j \text { at intersection } n \text { using } \\
\text { path } h_{a, b} \text { to connect origin } a \text { and destination } \\
b\end{array}$ \\
\hline$u_{n}:$ & Turn movement $u$ at intersection $n$ \\
\hline$v_{n}:$ & Turn movement $v$ at intersection $n$ \\
\hline$\omega_{n, u_{n}, v_{n}}:$ & $\begin{array}{l}\text { Clearance time (or Intergreen) at } \\
\text { intersection } n \text { separating a movement } u_{n} \text { and } \\
\text { another movement } v_{n}\end{array}$ \\
\hline$Q_{n, i, j}:$ & $\begin{array}{l}\text { Demand turning flow from } \operatorname{arm} i \text { to } \operatorname{arm} j \text { at } \\
\text { intersection } n\end{array}$ \\
\hline$q_{n, i, j, k}:$ & $\begin{array}{l}\text { Assigned lane flow from arm } i \text { to } \operatorname{arm} j \text { on } \\
\text { lane } k \text { at intersection } n\end{array}$ \\
\hline$s_{n, i, k}:$ & $\begin{array}{l}\text { Saturation flow on lane } k \text { from } \operatorname{arm} i \text { at } \\
\text { intersection } n\end{array}$ \\
\hline $\bar{s}_{n, i, k}$ & $\begin{array}{l}\text { Saturation flow on lane } k \text { from arm } i \text { at } \\
\text { intersection } n \text { for straight-ahead movement }\end{array}$ \\
\hline$r_{n, i, j, k}:$ & $\begin{array}{l}\text { Radius of a vehicle turning trajectory from } \\
\text { arm } i \text { to arm } j \text { on lane } k \text { at intersection } n\end{array}$ \\
\hline$f_{n, i, j, k}:$ & $\begin{array}{l}\text { Turning proportion of the assigned lane flow } \\
\text { from arm } i \text { to arm } j \text { on lane } k \text { at intersection } \\
n\end{array}$ \\
\hline$c:$ & Cycle length (cycle time in seconds) \\
\hline$\zeta:$ & Reciprocal of a cycle length $(=1 / c)$ \\
\hline$\theta_{n, i, j}:$ & $\begin{array}{l}\text { Start of green for all movements from arm } i \\
\text { to arm } j \text { on lane } k \text { at intersection } n\end{array}$ \\
\hline$\phi_{n, i, j}:$ & $\begin{array}{l}\text { Duration of green for all movements from } \\
\text { arm } i \text { to arm } j \text { on lane } k \text { at intersection } n\end{array}$ \\
\hline$\Theta_{n, i, k}:$ & $\begin{array}{l}\text { Start of green on lane } k \text { from } \operatorname{arm} i \text { at } \\
\text { intersection } n\end{array}$ \\
\hline$\Phi_{n, i, k}:$ & $\begin{array}{l}\text { Duration of green on lane } k \text { from arm } i \text { at } \\
\text { intersection } n\end{array}$ \\
\hline$g_{n, i, j}:$ & $\begin{array}{l}\text { Minimum duration of green for turn } \\
\text { movements from arm } i \text { to arm } j \text { at } \\
\text { intersection } n\end{array}$ \\
\hline$y_{n, i, k}:$ & $\begin{array}{l}\text { Flow factor on lane } k \text { from } \operatorname{arm} i \text { at } \\
\text { intersection } n\end{array}$ \\
\hline$p_{n, i, k}:$ & $\begin{array}{l}\text { Maximum degree of saturation on lane } k \\
\text { from } \operatorname{arm} i \text { at intersection } n\end{array}$ \\
\hline$\rho_{n, i, k}:$ & $\begin{array}{l}\text { Degree of saturation on lane } k \text { from } \operatorname{arm} i \text { at } \\
\text { intersection } n\end{array}$ \\
\hline$e:$ & $\begin{array}{l}\text { Difference between actual and effective } \\
\text { greens }\end{array}$ \\
\hline$\mu:$ & $\begin{array}{l}\text { Common flow multiplier to scale the given } \\
\text { OD demand flows }\end{array}$ \\
\hline$P_{a, b, h_{a, b}}:$ & $\begin{array}{l}\text { Path flow from origin } a \text { to destination } b \\
\text { using the } h_{a, b} \text { path. }\end{array}$ \\
\hline
\end{tabular}

\section{Conflicts of Interest}

The authors declare that there are no conflicts of interest regarding the publication of this paper.

\section{Acknowledgments}

The work described in this paper was supported by the GRF funding scheme from the Research Grants Council of the Hong Kong Special Administrative Region (Project no. CityU 123313).

\section{References}

[1] M. Q. Du, X. W. Jiang, and L. Cheng, "Estimating the capacity of urban transportation networks with an improved sensitivity based method," Discrete Dynamics in Nature and Society, vol. 2015, Article ID 827094, 13 pages, 2015.

[2] S.-W. Chiou, "An efficient algorithm for optimal design of area traffic control with network flows," Applied Mathematical Modelling, vol. 33, no. 6, pp. 2710-2722, 2009.

[3] Q. Meng, H. Yang, and M. G. H. Bell, "An equivalent continuously differentiable model and a locally convergent algorithm for the continuous network design problem," Transportation Research B: Methodological, vol. 35, no. 1, pp. 83-105, 2001.

[4] S. C. Wong and H. Yang, "Reserve capacity of a signal-controlled road network," Transportation Research Part B: Methodological, vol. 31, no. 5, pp. 397-402, 1997.

[5] H. Yang and M. G. H. Bell, "Models and algorithms for road network design: a review and some new developments," Transport Reviews, vol. 18, no. 3, pp. 257-278, 1998.

[6] S. C. Wong, C. Yang, and H. K. Lo, "A path-based traffic assignment algorithm based on the TRANSYT traffic model," Transportation Research, Part B: Methodological, vol. 35, no. 2, pp. 163-181, 2001.

[7] C. K. Wong, S. C. Wong, and H. K. Lo, "A spatial queuing approach to optimize coordinated signal settings to obviate gridlock in adjacent work zones," Journal of Advanced Transportation, vol. 44, no. 4, pp. 231-244, 2010.

[8] C. K. Wong and S. C. Wong, "A time-dependent lane-based cell transmission model for coordinated signal optimization," Transportation and Management Science, pp. 573-582, 2008.

[9] R. E. Allsop, "Evolving application of mathematical optimisation in design and operation of individual signal-controlled road junctions," in Mathematics in Transport and Planning and Control, J. D. Griffiths, Ed., pp. 1-24, Clarendon Press, Oxford, UK, 1992.

[10] R. A. Vincent, A. I. Mitchell, and D. I. Robertson, "User guide to TRANSYT version 8," Report LR888, Transport and Road Research Laboratory, Crowthorne, UK, 1980.

[11] M. R. Crabtree, “TRANSYT/9 Users' manual," Report AG8, Transport and Road Research Laboratory, Crowthorne, UK, 1988.

[12] N. H. Gartner, J. D. C. Little, and H. Gabbay, "Optimization of traffic signal settings by mixed-integer linear programming. Part I: the network coordination problem," Transportation Science, vol. 9, pp. 321-343, 1975.

[13] N. H. Gartner, J. D. C. Little, and H. Gabbay, "Optimization of traffic signal settings by mixed-integer linear programming. Part II: the network synchronization problem," Transportation Science, vol. 9, no. 4, pp. 344-363, 1975. 
[14] J. D. C. Little, N. H. Kelman, M. D. Kelman, and N. H. Gartner, "MAXBAND: a program for setting signals on arterials and triangular networks," Transportation Research Record, vol. 795, pp. 40-46, 1981.

[15] S. C. Wong, "Group-based optimisation of signal timings using the transyt traffic model," Transportation Research B: Methodological, vol. 30, no. 3, pp. 217-244, 1996.

[16] S. C. Wong and C. Yang, "An iterative group-based signal optimization scheme for traffic equilibrium networks," Journal of Advanced Transportation, vol. 33, no. 2, pp. 201-217, 1999.

[17] C. K. Wong and Y. Y. Lee, "Convergence study of minimizing the nonconvex total delay using the lane-based optimization method for signal-controlled junctions," Discrete Dynamics in Nature and Society, vol. 2012, Article ID 858731, 14 pages, 2012.

[18] C. Yu, W. Ma, H. K. Lo, and X. Yang, "Robust optimal lane allocation for isolated intersections," Computer-Aided Civil and Infrastructure Engineering, vol. 32, no. 1, pp. 72-86, 2017.

[19] C. K. Wong and S. C. Wong, "Lane-based optimization of signal timings for isolated junctions," Transportation Research Part B: Methodological, vol. 37, no. 1, pp. 63-84, 2003.

[20] C. K. Wong and S. C. Wong, "A lane-based optimization method for minimizing delay at isolated signal-controlled junctions," Journal of Mathematical Modelling and Algorithms, vol. 2, no. 4, pp. 379-406, 2003.

[21] C. K. Wong and B. G. Heydecker, "Optimal allocation of turns to lanes at an isolated signal-controlled junction," Transportation Research Part B: Methodological, vol. 45, no. 4, pp. 667-681, 2011.

[22] B. G. Heydecker, "Sequencing of traffic signals," in Mathematics in Transport and Planning and Control, J. D. Griffiths, Ed., pp. 57-67, Clarendon Press, Oxford, UK, 1992.

[23] R. M. Kimber, M. McDonald, and N. B. Hounsell, "Prediction of saturation flows for road junctions controlled by traffic signals," RR 67, Transport and Road Research Laboratory, Crowthorne, UK, 1986. 


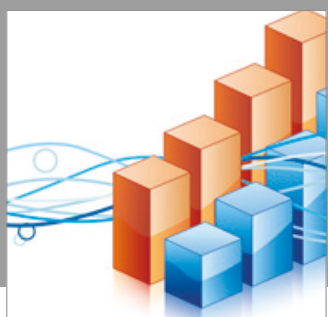

Advances in

Operations Research

vatersals

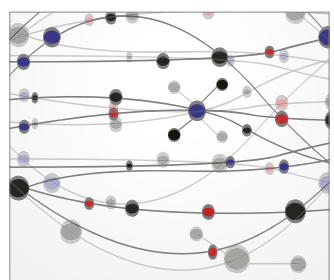

\section{The Scientific} World Journal
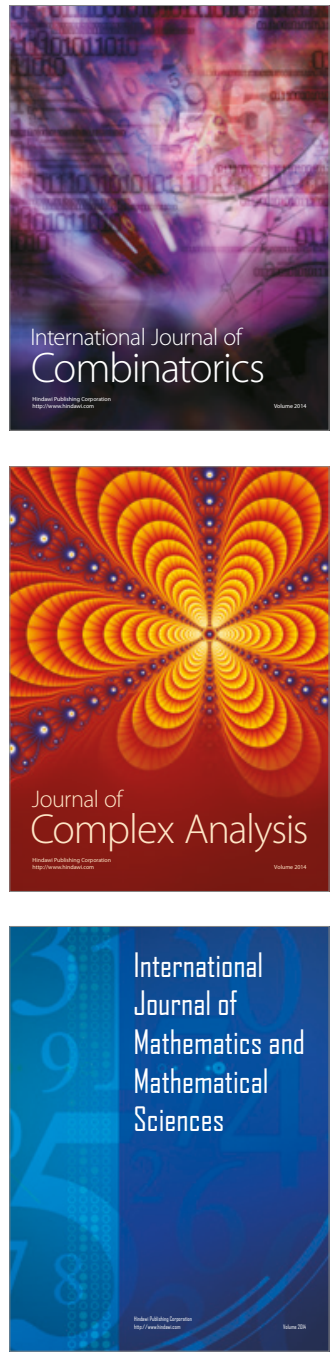
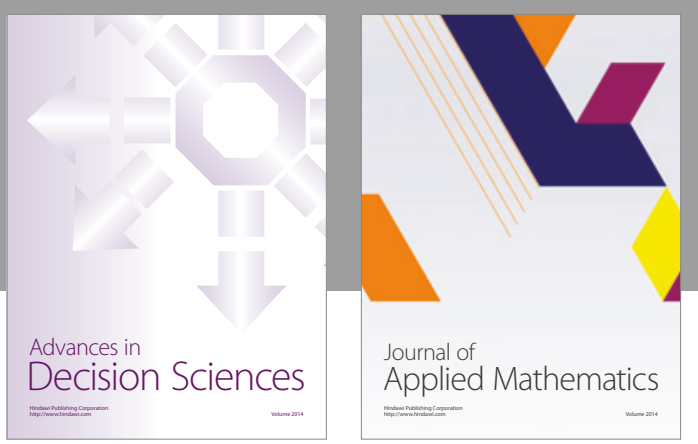

Algebra

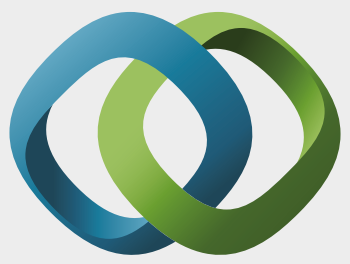

\section{Hindawi}

Submit your manuscripts at

https://www.hindawi.com
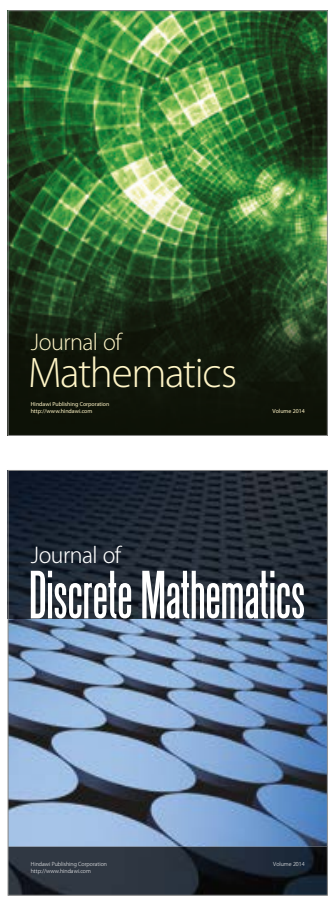

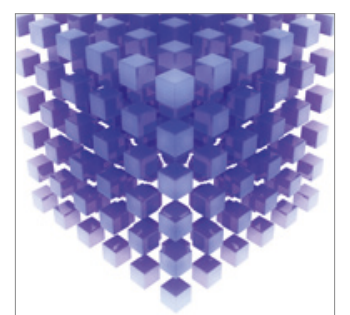

Mathematical Problems in Engineering
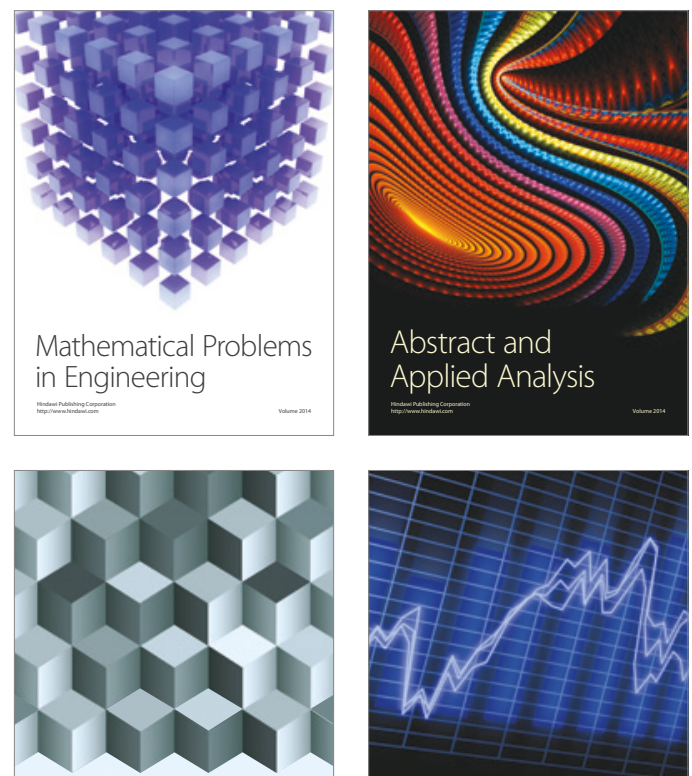

Journal of

Function Spaces

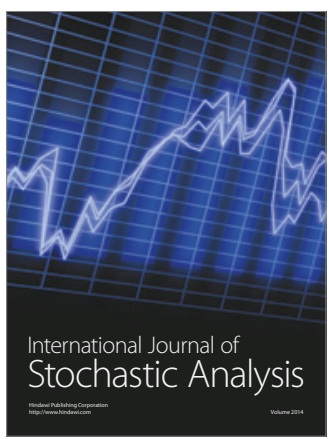

Probability and Statistics
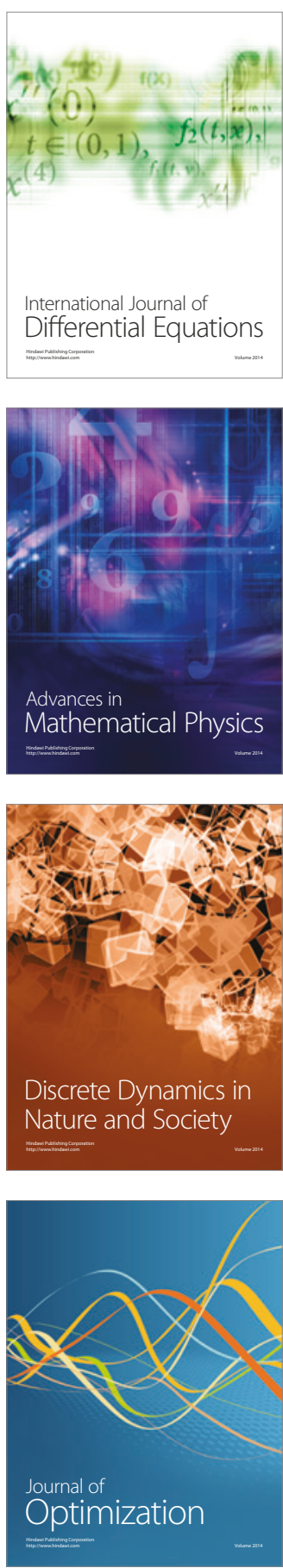